\title{
AIAA 2001-3337 \\ Ground Simulations of Electron \\ Current to Wide Probes for Electrodynamic Tethers
}

Brian E. Gilchrist

University of Michigan, Ann Arbor, Michigan 48109

Sven G. Bilén

The Pennsylvania State University, University Park,

Pennsylvania 16802

Éric Choinière, Alec D. Gallimore, and Daniel A. Herman

University of Michigan, Ann Arbor, Michigan 48109

\section{7th AIAA/ASME/SAE/ASEE Joint Propulsion Conference and Exhibit$$
\text { 8-11 July } 2001
$$$$
\text { Salt Lake City, Utah }
$$

For permission to copy or republish, contact the American Institute of Aeronautics and Astronautics 1801 Alexander Bell Drive, Suite 500, Reston, VA 20191-4344 


\title{
Ground Simulations of Electron Current to Wide Probes for Electrodynamic Tethers
}

\author{
Brian E. Gilchrist* \\ University of Michigan, Ann Arbor, Michigan 48109 \\ Sven G. Bilén ${ }^{\dagger}$ \\ The Pennsylvania State University, University Park, Pennsylvania 16802 \\ Éric Choinière \\ University of Michigan, Ann Arbor, Michigan 48109
}

\begin{abstract}
We describe chamber tests of simulated electrodynamic tethers (EDTs) of different geometries operating in a dense, high-speed plasma. The geometries tested and described here were cylindrical and flat-ribbon. Several important conclusions that can be drawn from the tests are as follows: the currents collected by cylinder are close to what would be predicted via orbital-motion-limited (OML) current collection theory. The tape tether had comparable current levels to a theoretical equal area OML cylinder collector. However, I-V behavior clearly is different at nearest distances ( $\sim 16 \lambda_{D}$ tape width) as compared to furthest test distances $\left(\sim 6 \lambda_{D}\right.$ tape width). The tape tether did better than a theoretical equal mass solid cylinder. A "knee" in the I-V curves can be seen in the tape data at a potential that is near the estimated energy of the incoming beam of ions, at least for the closest distances where Debye length is smallest. Below this knee the current increases rapidly as voltage is increased. Above the knee the current increases at a rate near that expected from OML current-collection models depending on the relative width. This likely is an example of high-speed plasma flow effect. Perpendicular tape orientation performed slightly better than parallel.
\end{abstract}

\section{Nomenclature}

$A_{p} \quad$ probe surface area, $\mathrm{m}^{2}$

$d \quad$ wire diameter, $\mathrm{m}$

$F$ thrust, $\mathrm{N}$

$g$

$I_{d}$

$I_{E}$

$I_{p}$

$I_{\mathrm{sp}}$

$k$

$K$

$l$

electron mass, $9.109 \times 10^{-31} \mathrm{~kg}$

$m_{i} \quad$ ion mass, $\mathrm{kg}$

$n_{e} \quad$ electron plasma density, $\mathrm{m}^{-3}$

$P$ power, $\mathrm{W}$

$q \quad$ charge magnitude, $1.602 \times 10^{-19} \mathrm{C}$

$r \quad$ wire radius, $m$

$t \quad$ tape thickness, $\mathrm{m}$

$T$ wire temperature, $\mathrm{K}$

*Associate Professor, Electrical Engineering and Space Systems, AIAA Senior Member

${ }^{\dagger}$ Assistant Professor, Cornmunications and Space Sciences Laboratory, AIAA Member

$\ddagger$ Graduate Student, Radiation Laboratory, EECS Department

$\S_{\text {Associate Professor, Aerospace Engineering and Applied }}$ Physics, AIAA Associate Fellow

Graduate Student, Aerospace Engineering

Copyright (c) 2001 by Brian E. Gilchrist. Putlisted by the American Institute of Aeronautics and Astrogautics, Inc. with permission.
$T_{e} \quad$ electron temperature, $\mathrm{K}$

$V_{a} \quad$ accelerating potential, $\mathrm{V}$

$V_{d} \quad$ thruster discharge voltage, $\mathrm{V}$

$V_{l} \quad$ thruster loss voltage, $\mathrm{V}$

$v_{t e} \quad$ electron thermal velocity, $\mathrm{m} / \mathrm{s}$

$w \quad$ tape width, $\mathrm{m}$

$\beta \quad$ ration of measured currents

$\varepsilon \quad$ spectral emissivity

$\varepsilon_{0} \quad$ free space permittivity, $8.85 \times 10^{-12} \mathrm{~F} / \mathrm{m}$

$\eta \quad$ discharge electron current fraction

$\lambda_{D} \quad$ Debye length, $\mathrm{m}$

$\rho$ resistivity, $\Omega \cdot \mathrm{cm}$

$\sigma \quad$ Stefan-Boltzmann const., $5.67 \times 10^{-8} \mathrm{~W} / \mathrm{m}^{2} \mathrm{~K}^{4}$

\section{Introduction}

T $\mathrm{T}$ has been proposed that operating in the orbital1 motion-limited (OML) regime is especially beneficial for electron current collection to thin, bare electrodynamic tethers (EDTs) with width dimensions on the order of a Debye length. ${ }^{1}$ This configuration is different than that of Tethered Satellite System missions (TSS-1 and -1R), which used a large (with respect to $\lambda_{D}$ ) spherical collector for electron collection. It is predicted theoretically that a bare tether will be a highly efficient collector of ionospheric electrons (per unit area) when compared to other current collection geometries at equal bias (such as TSS) and it also has been suggested that a combination of technologies may be useful. 
As part of NASA's Advanced Space Transportation Program, the Propulsive Small Expendable Deployer System (ProSEDS) mission, ${ }^{2}$ which is to fly in 2002, will be the first to use the bare-tether concept and will demonstrate high current and measurable thrust; the technology is also being considered for other future missions. However, a small, thin cylinder is not necessarily the best tether design when considering other practical factors such as tether lifetime. For example, to increase tether lifetime, a tether based on ribbonlike geometry (e.g., flat and wide) or more sparse structures of equal series resistance may be preferred; hence, these tethers would have dimensions exceeding a Debye length. These new geometries pose several questions that must be addressed before they can be employed. For example, how will the current collection performance change as a function of geometry and Debye length when in an ionospheric plasma at orbital velocities? Does the orientation of the ribbon with respect to the flowing plasma direction have an impact on collection efficiency?

This work describes two sets of vacuum chamber tests that simulated EDTs of different geometries operating in a dense, high-speed plasma. The geometries described here were cylindrical and flat-ribbon tape. The cylinder sample had a radius ranging 1-3 Debye lengths (as plasma parameters were varied) whereas the tape had a width that ranged from 6-16 Debye lengths. For these tests, the 6-m $\times 9-\mathrm{m}$ chamber operated by Michigan's Plasmadynamics and Electric Propulsion Laboratory (PEPL) was used along with a PEPL/USAF-designed Hall thruster for the plasma source. These tests were done, in part, to support design efforts for the follow-on mission to ProSEDS called STEP-Airseds, which will demonstrate multiple boost/deboost and inclination-change operations over a period of a year and covering from below $400-\mathrm{km}$ to above $700-\mathrm{km}$ altitude.

\section{OML Current Collection}

In the OML regime, the current, $i$, collected by a conductor in non-flowing plasma is given by the equation

$$
i=n_{e} A_{p} q \frac{\sqrt{2}}{\pi} v_{t e}\left(1+\frac{q V}{k T_{e}}\right)^{0.5}
$$

The thermal velocity here is given by $v_{t e}=\sqrt{k T_{e} / m_{e}}$. For high potentials $\left(V \gg k T_{e} / q\right)$, Eqn. 1 reduces to

$$
i=n_{e} A_{p} q \frac{\sqrt{2}}{\pi}\left(\frac{q V}{m_{e}}\right)^{0.5} .
$$

We see that for large potentials, the current collected is independent of electron temperature and scales as the square root of the potential.

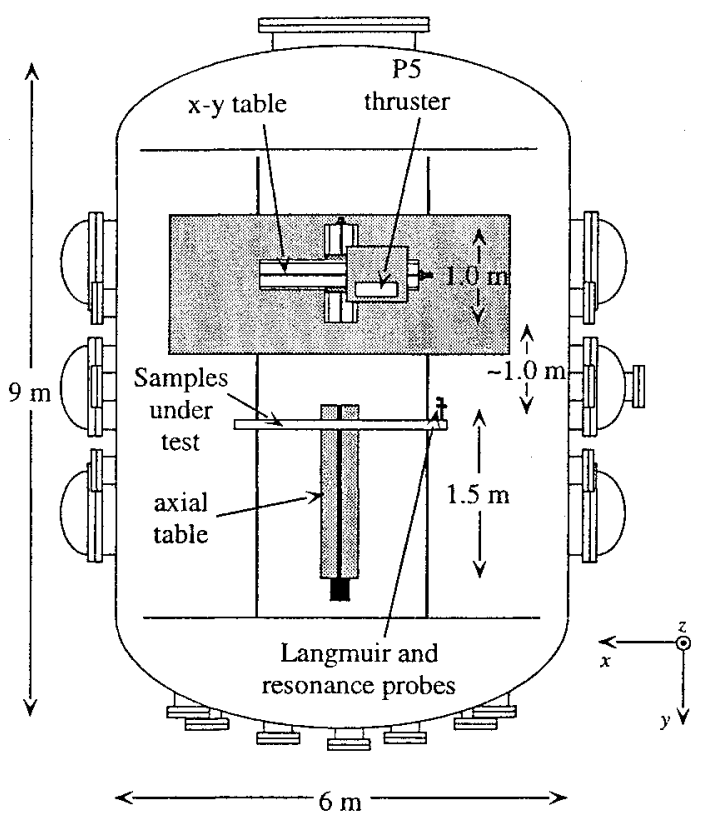

Fig. 1 PEPL LVTF as configured for tests.

Chamber Description and Setup

\section{Experimental Setup}

PEPL has as its centerpiece the Large Vacuum Test Facility (LVTF), a cylindrical, stainless-steel-clad tank that is $9 \mathrm{~m}$ long and $6 \mathrm{~m}$ in diameter. ${ }^{4}$ The facility has seven nude cryopumps backed by two $2000 \mathrm{cfm}(56,600$ $l / \mathrm{s})$ blowers and four $400 \mathrm{cfm}(11,300 \mathrm{l} / \mathrm{s})$ mechanical pumps. These pumps give the facility a combined pumping speed of $500,000 \mathrm{l} / \mathrm{s}$ on air and $240,000 \mathrm{l} / \mathrm{s}$ on xenon and provide the ability to reach a high vacuum ( $\sim 10^{-7}$ torr). Fig. 1 is a diagram of the LVTF as it was set up for these tests.

Two positioning tables were used to change the separation distance between the thruster and sample plane and to locate the sample under test (SUT) directly along the thruster's centerline. The thruster was mounted on an $x-y$ table that could move axially $1.0 \mathrm{~m}$ and radially sufficient to cover all samples. The samples were mounted on an aluminum frame that was connected to an axial table that could move $1.5 \mathrm{~m}$ axially (samples are described in more detail below). Combined table movement allowed thrustersample separation distance to change from $\sim 1 \mathrm{~m}$ to $\sim 3 \mathrm{~m}$. Changing separation distance was the primary mechanism for changing the plasma density seen at the sample plane.

\section{Samples}

Tungsten metal was used for all samples to ensure that they would endure the expected high temperatures, which were caused by the collection of highenergy electrons to the samples' surfaces. The rational 


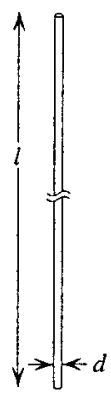

(a)

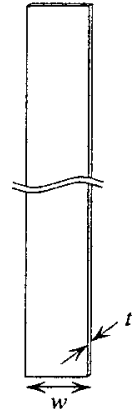

(b)
Fig. 2 Schematics of samples used for both test sets: (a) reference cylinder, (b) tape.

for and the implications of the use of tungsten are discussed below. During the first set of tests, the samples were approximately $10 \mathrm{~cm}$ in length, whereas during the second set of tests, the samples were approximately $30 \mathrm{~cm}$ in length. Two tape samples were used in both test sets, one where the width dimension was perpendicular to the plasma flow, the other with the width dimension parallel to the plasma flow. The thickness of the tape was $t=0.1 \mathrm{~mm}$. Schematic representation of the samples is given in Fig. 2. Sample descriptions are summarized in Table 1.

All samples were soldered to the center conductor of a bulkhead SHV (safe high voltage) connector; the connector-sample interface was then covered with vacuum epoxy. This sample assembly was then mounted in a piece of stainless-steel flashing, which also functioned as the sample mounting plate. These mounting plates served as localized ground planes and were themselves electrically connected to the sample support frame. For both test sets there existed a minimum of $10-\mathrm{cm}$ clearance between the samples and the frame. A picture of the setup is shown in Fig. 3 .

Use of Tungsten for Samples

Due to the energetic electron bombardment of the samples under high potentials, the samples would tend to heat up very quickly, and probably exceed the melting temperature of most metals. Tungsten was therefore used for all samples because it has a melting temperature of $3695 \mathrm{~K}$, which is significantly higher than other metals with low resistivity, such as copper, silver, or gold. Tungsten has a resistivity of $\rho=5.51 \times 10^{-6} \Omega \cdot \mathrm{cm}$, which is within a factor of $2-3$ of these other metals.

By performing the following qualitative analysis, we can determine expected worst-case temperatures for the tungsten during current collection. First, we assume the probe is thermally isolated, the surface is equipotential, and finally assume that all electrons collected at the surface (i.e., the electron current to the

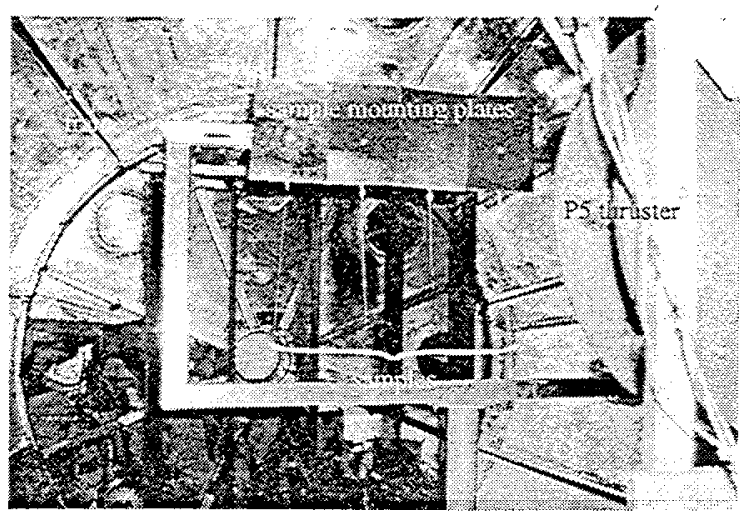

Fig. 3 Picture of the sample support structure for the second test set; structure for the first test set was very similar.

sample) have been accelerated to the potential of the sample. Then, the power heating the sample is simply $P\left(V_{a}\right)=I\left(V_{a}\right) V_{a}$, where $V_{a}$ is the accelerating potential and the current is a function of $V_{a}$. Fortunately, as the sample's temperature increases, so does its spectral emissivity, $\varepsilon$, which slows down runaway heating slightly. (The electron emission will also increase, but we will ignore this in this analysis.) Using StefanBoltzmann's Law, we find the temperature, $T$, of the wire to be

$$
T\left(V_{a}\right)=\left(\frac{P\left(V_{a}\right)}{\sigma A \varepsilon}\right)^{0.25},
$$

From this, we see that $T$ is a function of $V_{a}$. We also note that since $P$ is linearly dependent on $A$ in the OML collection regime, and $A$ is found in the denominator of the right-hand side of Eqn. 3 , that surface area drops out of the equation. What this means is that you are no better or worse off by increasing or decreasing the surface area. Calculations show that at potentials above $\sim 300-400 \mathrm{~V}$ for the plasma densities of these tests, the tungsten is in danger of melting. Hence, a second temperature mitigation method is needed if potentials higher than these values are used. By pulsing voltages at a low duty cycle, the sample is given a chance to cool off before the next pulse of high voltage heats it. The on-time must be short because the heating occurs quickly, on the order of 10 s of milliseconds.

\section{Thruster Description and Operating Conditions}

The PEPL/AFRL "P5" 5-kW-class Hall-effect thruster was used to provide a flowing plasma for these experiments. A detailed description of the P5 is given by Haas et al..$^{5}$ and a picture of this thruster is shown in Fig. 4. When operating nominally, the P5 plasma is too fast and dense for the conditions we needed; hence, for these tests the thruster was set at off-nominal conditions in order to lower the plasma velocity and density seen along the thruster's axial direction. The P5 
Table 1 Descriptions of samples used for both test sets.

\begin{tabular}{||rl||l|l|l||}
\hline \hline \multicolumn{1}{||c||}{ Sample } & $\mathbf{2}$ & $\mathbf{2}$ & $\mathbf{3}$ \\
\hline \multirow{2}{*}{ Description } & $\begin{array}{l}\text { Reference } \\
\text { Cylinder }\end{array}$ & $\begin{array}{l}\text { Perpendicular } \\
\text { Tape }\end{array}$ & $\begin{array}{l}\text { Parallel } \\
\text { Tape }\end{array}$ \\
\hline first & length, $l(\mathrm{~cm})$ & 10.0 & 9.4 & 9.2 \\
\cline { 2 - 5 } set & Width, $w$, or & 0.28 & 1.9 (top) to & 2.0 (top) to \\
Diameter, $d(\mathrm{~mm})$ & & 2.1 (bottom) & 2.2 (bottom) \\
\hline second & length, $l(\mathrm{~cm})$ & 29.5 & 29.5 & 29.5 \\
\cline { 2 - 5 } test & Width, $w$, or & 0.28 & 2.0 (top) to & 2.0 (top) to \\
set & Diameter, $d(\mathrm{~mm})$ & & 2.4 (bottom) & 2.3 (bottom) \\
\hline
\end{tabular}

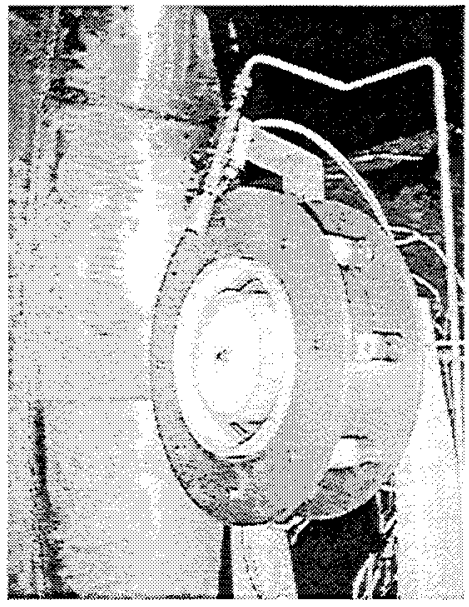

Fig. 4 P5 Thruster.

operating conditions for both sets of tests are given in Table 2. For these experiments, the thruster was run on xenon propellant $\left(m_{i}=2.18 \times 10^{-25} \mathrm{~kg}\right)$. The P5 operating conditions for both sets of tests are given in Table 2.

At the time of the tests, the emitted beam energy could only be estimated ( $\sim 20-50 \mathrm{eV}$ assuming a 100 $\mathrm{V}$ discharge voltage) since the discharge voltage was below the nominal thruster operating range and we did not have sensors available to help resolve the character of the P5 flow. Recently, additional tests have allowed us to better estimate bulk and near-field ion beam velocity for the 'Table 2 conditions.

Bulk velocity measurements were made by measuring the thrust and mass flow rate to characterize bulk plasma drift velocity of the P5 engine. The bulk drift velocity is simply the thrust divided by the anode propellant flow rate (the cathode flow is neglected as it makes no direct contribution to the acceleration of the plasma). Appendix A gives a brief description of the measurement technique. For the first test set, an estimated average bulk directed energy was $14 \mathrm{eV}( \pm 2$ $\mathrm{eV}$ or better) and for the second test set it was $21 \mathrm{eV}$ $( \pm 2 \mathrm{eV}$ or better).

Laser induced fluorescence (LIF) measurements also were made recently out to a distance of $75 \mathrm{~cm}$ from the thruster exit plane for the conditions indicated in Table 2. However, as of this writing, data analysis has not been completed except to note that signal quality appeared good. The LIF measurements will allow us to obtain not only the primary velocity, but also a distribution function relation for multiple velocities.

We mention at this point several issues regarding the use of the P5 thruster as our plasma source. First, since the flow character off axis from the P5 could not be resolved at the time of the tests, an assumption of a uniform flow along the full length of the test probe may not be valid. However, this was partially compensated for by placing each sample in the same location with respect to the thruster at a given distance. Second, we were not able to measure the population of slowmoving ions. This also may become an issue because of the possibility for charge exchange between an accelerated ion and slow-moving neutrals. This will be partially addressed when the LIF data is fully analyzed, at least out to $75 \mathrm{~cm}$ from the thruster. The P5 plasma environment as measured during these tests is presented below.

\section{Data Collection and Measurement System Current-Voltage Measurement System}

A schematic of the current-voltage measurement system is given in Fig. 5. The system consisted of a Universal Voltronics BRC 20,000 HV power supply connected to the tether samples by way of a switch box inside the chamber. The HV power supply was controlled via RS- 232 by the computer controller running a custom virtual instrument (VI) under LabVIEW. The computer commanded the HV supply to a specified voltage and then quickly back to zero (within 50-100 ms) to minimize sample heating. Before returning to zero, the supply's internal voltage sensor reported back the actual voltage obtained. We verified that the supply pulses were long enough such that the voltage had reached a steady-state value. An American Aerospace Controls 835-2-10 current sensor (ammeter) was used to measure current. To increase sensitivity, the HV supply line was looped through the sensor 10 times. Since the output of the ammeter was a voltage signal, an HP $34401 \mathrm{~A}$ voltmeter was used to

$$
4 \text { of } 10
$$


Table 2 Thruster operating parameters for both test sets.

\begin{tabular}{|l||c|c||}
\hline \hline Parameter & First Test Set & Second Test Set \\
\hline \hline Chamber Pressure (Gauge 1) & $1 \times 10^{-5}$ torr & $6.8 \times 10^{-6}$ torr \\
Chamber Pressure $(G a u g e ~ 2)$ & $2 \times 10^{-5}$ torr & $1.5 \times 10^{-5}$ torr \\
Discharge Voltage $\left(V_{d}\right)$ & $100 \mathrm{~V}$ & $100 \mathrm{~V}$ \\
Discharge Current $\left(I_{d}\right)$ & $5.3 \mathrm{~A}$ & $4 \mathrm{~A}$ \\
Inner Magnet Current $\left(I_{\mathrm{im}}\right)$ & $2.99 \mathrm{~A}$ & $1.04 \mathrm{~A}$ \\
Outer Magnet Current $\left(I_{\mathrm{im}}\right)$ & $1.99 \mathrm{~A}$ & $1.03 \mathrm{~A}$ \\
Cathode Voltage $\left(V_{c}\right)$ & $-19.7 \mathrm{~V}$ & $-19.2 \mathrm{~V}$ \\
Heater Voltage $\left(V_{\mathrm{htr}}\right)$ & $7.1 \mathrm{~V}$ & $6.0 \mathrm{~V}$ \\
Anode Flowrate $\left(\dot{m}_{a}\right)$ & $60 \mathrm{sccm}$ & $45 \mathrm{sccm}$ \\
Cathode Flowrate $\left(\dot{m}_{c}\right)$ & $6 \mathrm{sccm}$ & $6 \mathrm{sccm}$ \\
\hline
\end{tabular}

measure it and, in conjunction with the voltage measurement described above, these values were recorded as voltage-current pairs.

\section{Plasma Parameter Measurements}

Three separate probes of two different types were used to measure the plasma environment. Two Langmuir probes (LPS) were mounted as follows: one parallel to the plasma flow and the other perpendicular to the flow. The LP sweeps were made by a Keithley 2410 source electrometer and collected via a custom VI by a computer controller (Fig. 5). A resonance probe (RP) was also co-located with the LPs. The resonance probe has been used previously to measure plasma densities in the plume of the P5 thruster. ${ }^{6}$ Although still an experimental technique, the RP yields good estimates of density in the far-field region of the P5 plume.

\section{Data}

In order to better understand the data presented here, we present first the plasma environment for both test sets and then the data collected during those tests.

\section{Plasma Environment}

Fig. 6 reports the measured plasma environment as a function of distance for both test sets. By positioning the sample plane closer and farther from the thruster, we were able to obtain approximately a factor-of-10 change in plasma density. There are several qualifications that must be mentioned concerning the determination of the plasma environment. First, LP data were successfully gathered and analyzed for the first round of tests, but RP data were not collected. The LP data from both the horizontal and vertical probes, which require different analyses due to their orientations, were compared and agreed to within a factor of 2 . Unfortunately, during the second test set, the LP data could not be trusted. Hence, we utilized RP data exclusively for the $187-, 230-$, and $305-\mathrm{cm}$ positions. However, we had to estimate the $100-\mathrm{cm}$ position from the OML current fits. Because the RP does not provide $T_{e}$ measurements, we were unable to explicitly measure $T_{e}$ for the second test set, but in comparison with data from the first test set, have set $T_{e}=0.8 \mathrm{eV}$ for all positions.

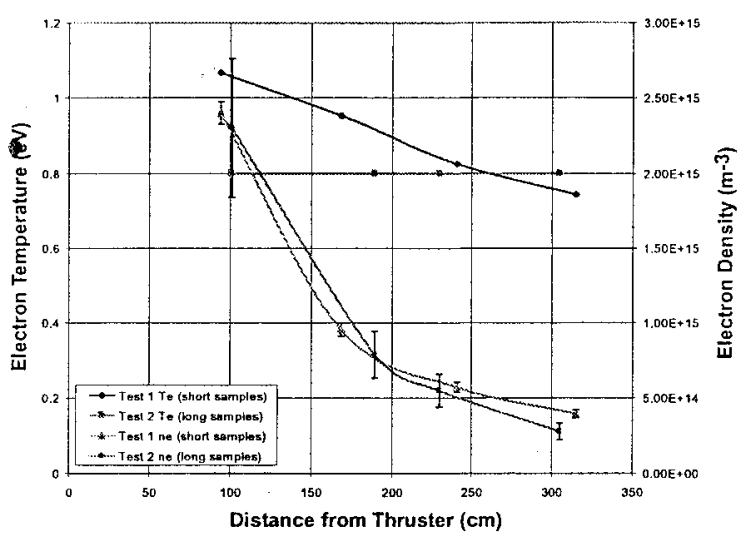

Fig. 6 Plasma parameters measured for both test sets.

Having said this, we feel that we were able to provide an adequate description of the plasma environment for all tests.

Fig. 7 shows the calculated Debye lengths for both test sets. Debye length is given by the following equation

$$
\lambda_{D}=\sqrt{\frac{\varepsilon_{0} k T_{e}}{q^{2} n_{e}}} .
$$

The cylindrical sample had a radius $r=d / 2=0.14$ $\mathrm{mm}$, so we can see the Debye lengths obtained spanned a range of $\sim 1-3$ times the cylinder's radius. The tape width of $\sim 2-2.4 \mathrm{~mm}$ represents from $5-16$ times the Debye length.

\section{Current Collection Data}

This first set of figures (Figs. 8a-d) plots the current as a function of voltage for the tape and cylinder geometries from the second test set at separation distances of $305,230,187$, and $100 \mathrm{~cm}$, respectively, which represents lower to higher plasma density (see Fig. 6). They also include the calculated OML currents to equal area equivalent cylinders (the tape equivalent cylinder radius is approximately $7 \mathrm{~mm}$ ). As the separation distance is reduced, the effective width of the 


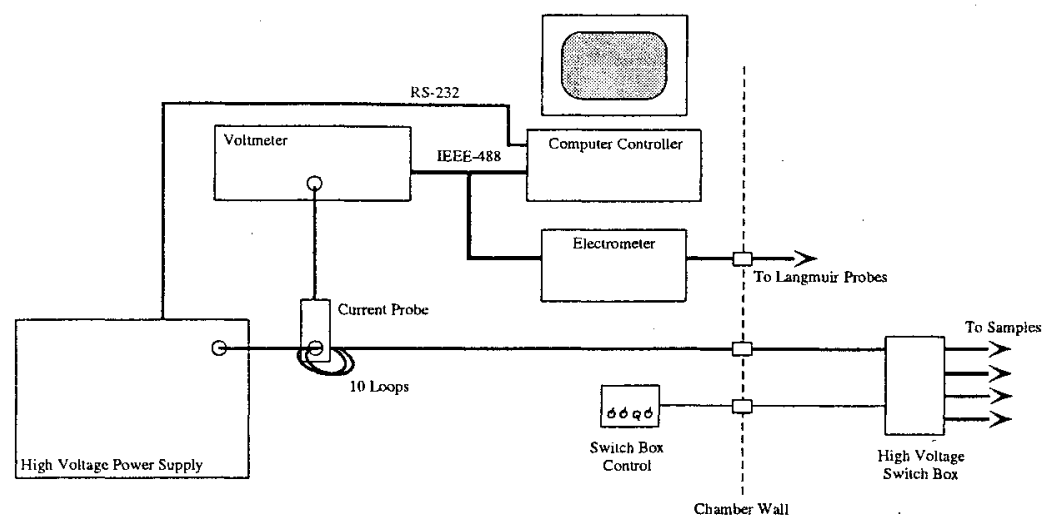

Fig. 5 Schematic of the test equipment setup.

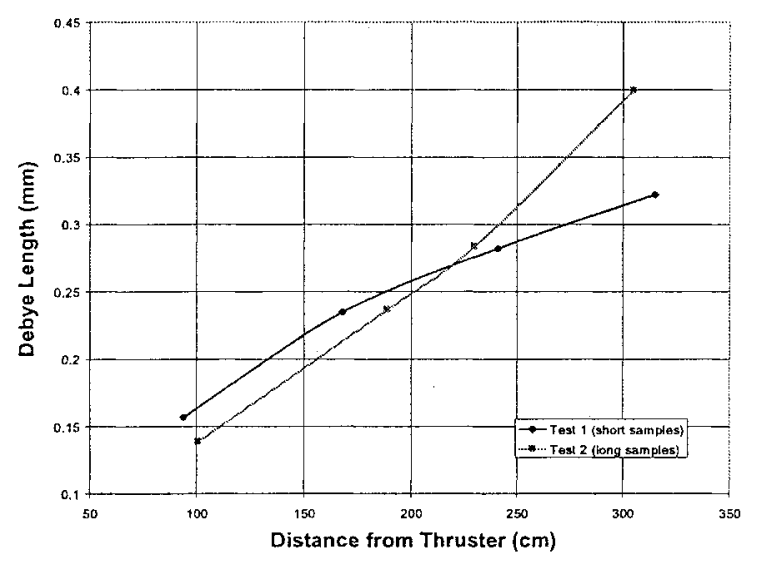

Fig. 7 Debye lengths derived for both test sets.

probes in terms of $\lambda_{D}$ increases. In general, the calculated model response was near the experimental data. However, the data has important differences in features that will be discussed below. It is also noted that there is some difference between parallel and perpendicular tape orientations.

Fig. 9 provides an example of differences between tests 1 and 2. To compare the differences between the two test runs, given the factor-of-three difference in sample length, the currents obtained in test 1 were multiplied by three before plotting. As seen in the figure, the cylinder samples were approximately the same, however, there is significant difference between the tape tethers. This is thought to be due to sample end-effects and is discussed below.

\section{Observations and Analysis}

There are several interesting and intriguing observations that result from the data collected during these tests and presented above. From Fig. 8 it can be seen that there is a small, but definite, difference between parallel and perpendicular orientations of the tape tether. In the figure it can be seen that the perpendicular orientation consistently exceeded the collected current of the parallel orientation and the difference appears to grow at higher densities (shorter Debye lengths). In all cases (different plasma densities), the onset of the separation between curves for the different orientations occurred near a threshold of $60-\mathrm{V}$ bias and was independent of current magnitude. In all but the $100-\mathrm{cm}$ case the difference in current for the orientations appears to reach a constant value that is maintained over the remaining potential range. The $100-\mathrm{cm}$ case appears to show the two curves converging.

We can also describe this separation between the parallel and perpendicular tape orientations in terms of a "knee" in the I-V curves for the parallel case that is most pronounced at higher densities (closer distances). A separate knee is also evident at least for the $100-\mathrm{cm}$ perpendicular tape case at a higher potential around $100 \mathrm{~V}$. At potentials below this knee, the current increases rapidly as potential is increased, but not quite as rapid as the OML model. Above the knee for the 305-, 230-, and 187-cm cases, the current increases at a rate that approaches the OML current collection model. For the 100-cm case, the collected current appears to follow a shallower slope than predicted from OML theory.

It is interesting to note that a change in current collection behavior at a bias threshold was also seen in the TSS $-1 \mathrm{R}^{9}$ and TSS -1 missions ${ }^{10}$ where the threshold was at a potential of the ionospheric ion beam energy. In these situations, the presence of heating and turbulence could be detected in the plasma. when the ion beam could be reflected from the collecting spacecraft. ${ }^{8}$ We note that the knee in our case, occurring near $60 \mathrm{~V}$, is somewhat above the estimated energy of the incoming beam of ions $(\sim 21 \mathrm{~V})$. Additional measurements may be required to determine 


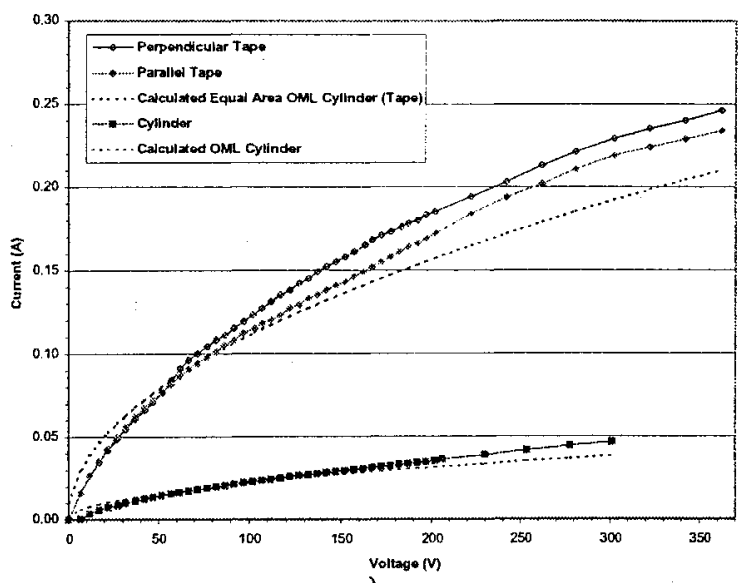

a)

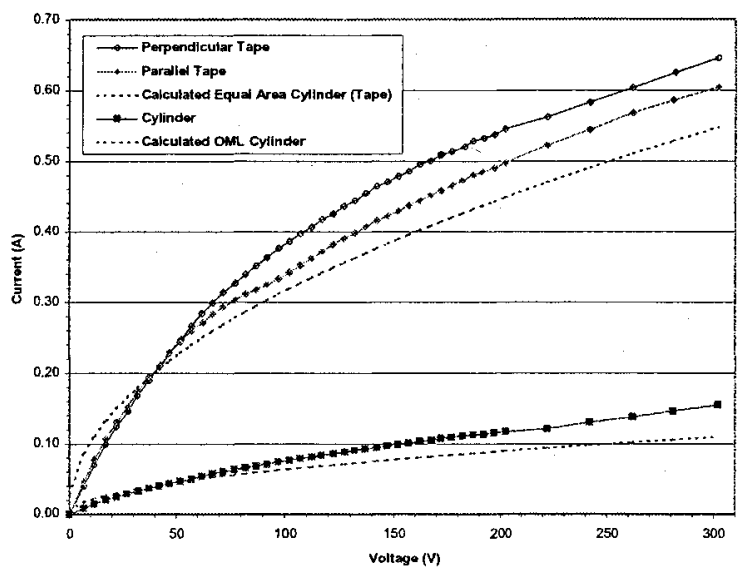

c)

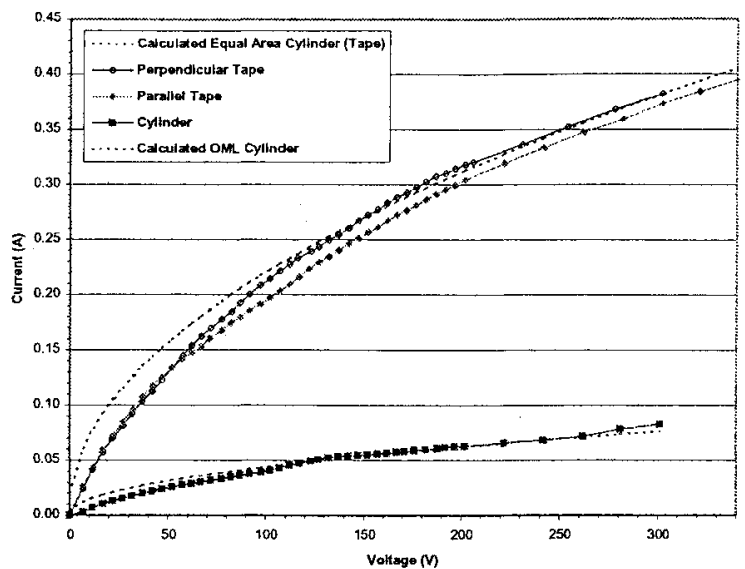

b)

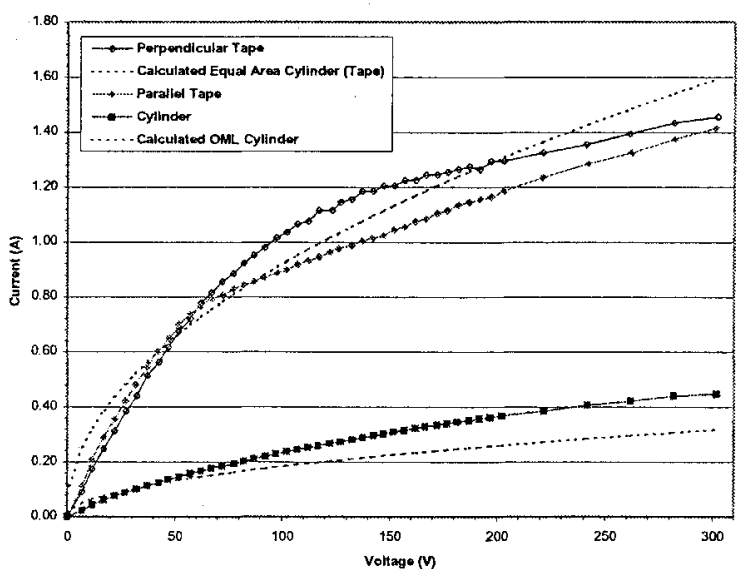

d)

Fig. 8 Current as a function of voltage for the tape and cylinder geometries from the second test set at separation distances of (a) $305 \mathrm{~cm}$, (b) $230 \mathrm{~cm}$, (c) $187 \mathrm{~cm}$, and (d) $100 \mathrm{~cm}$.

what effects are causing our knee response and which are independent of plasma density. Further, it will be shown below in Fig. 9 that even a change in ion beam energy from 14 (Text case 1) to $21 \mathrm{eV}$ (Test case 2) does not affect the $60-\mathrm{V}$ threshold.

We find it also significant that an equal-area OML cylinder model closely agrees with the prediction of collected current as seen for the tape samples even though the width of the tape is much larger than a Debye length (see Fig. 8). (Although, we note it is also possible that OML model results assume a constant density over the entire length of the probe. If the density is actually falling off away from the mid-point of the probe then the model would overpredict current collection.) Sanmartín and Estes ${ }^{11}$ considered tape geometries in the OML regime of a stationary, unmagnetized plasma and concluded that there are dimensions beyond a Debye length, for conditions that depend on potential and plasma temperatures, where a thin tape could satisfy OML collection. Their conclusions for the case appropriate to our experiments would suggest that the tape width could be up to four Debye lengths and still satisfy OML collection. Recently reported experimental work by Kruijff et al. ${ }^{13}$ show similar findings. Clearly, we have significantly exceeded this dimension here for our moving plasma. However, a more recent paper by Estes and Sanmartín ${ }^{12}$ suggests that their earlier threshold predictions are weak, i.e., degradation in performance is not rapid beyond the theoretical limits. This certainly seems to be the case here. Further, the response at $100 \mathrm{~cm}$ where the tape is $\sim 15 \lambda_{D}$ wide and where the current appears to be following a shallower slope may be indicative of exceeding the OML limit. Again, additional experiments will be required to answer this more clearly as it is also possible that we have reached a current level that exceeds what can easily be drawn from the thruster $(1.4 \mathrm{~A} \mathrm{col-}$ lected to probe with about $80 \%$ of the $4-\mathrm{A}$ discharge 
current for Test Case 2 available in the plasma).

Fig. 9 compares our first and second test runs for the tape and cylinder cases at 94 and $100 \mathrm{~cm}$. Here, the Test 1 current has been multiplied by three to crudely compensate for the fact that the samples were three times shorter than the Test 2 case. We attribute the difference in the tape results as a result of an end-effect even though the Test- 1 samples were over $600 \lambda_{D}$ long (end-effects are also multiplied by this same factor of three). In fact, a major reason why the Test- 2 run was undertaken was to lengthen the probe samples such that it more completely covered the $\mathrm{P} 5$ plume region to minimize end effects.

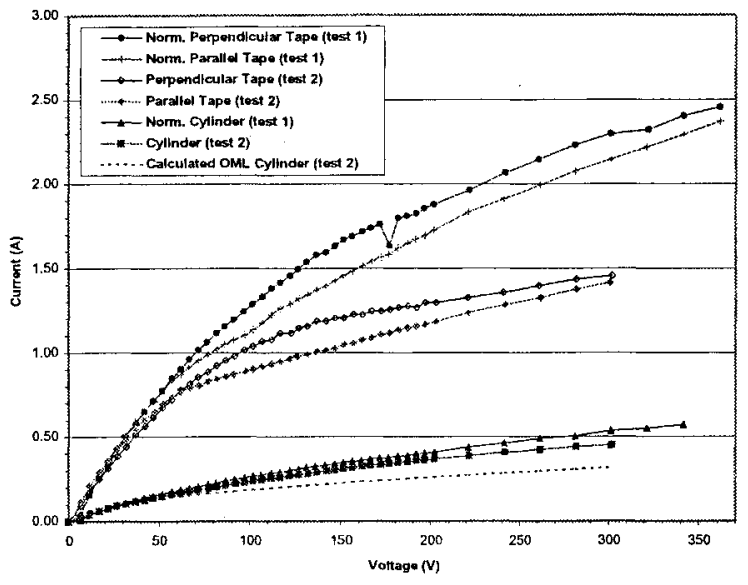

Fig. 9 Comparison of tests 1 and 2.

End effects are well known and they are often modeled by an ideal cylinder with an ideal spherical collector at the end, but the effective radius of the spherical collector is found to be much wider than probe radius ${ }^{14}$ and thus hard to easily model. However, because we have data sets for two different probe lengths, but roughly the same end effects for a given bias potential (this requires the plasma density is the same at the ends of the probe for both data sets), it is possible to quantitatively assess the presence of end effects.

We begin by noting that the voltage dependence of a infinite cylindrical probe would have a $V_{a}^{0.5}$ dependence with bias voltage. A spherical collector would have a $V_{a}^{1.0}$ relation. We define the ratio of end effect current to cylindrical probe current as either $K_{10}$ or $K_{30}$ for the $10-\mathrm{cm}$ or $30 \mathrm{-cm}$ probes, respectively (test case 1 and 2, respectively) and use as follows:

$$
\begin{aligned}
I_{10} & =I_{p 10}+I_{E}=I_{p 10}\left(1+K_{10}\right) \\
I_{30} & =I_{p 30}+I_{E}=I_{p 30}\left(1+K_{30}\right) \\
& =3 I_{p 30}\left(1+\frac{K_{10}}{3}\right)
\end{aligned}
$$

where $I_{10}$ and $I_{30}$ are the measured total currents for the two cases at a given bias potential, $I_{p x x}$ is the portion of the total current from an ideal cylindrical probe response, and $I_{E}$ is the end effect current assumed to be the same for both probes. We define an additional parameter, $\beta=3 I_{10} / I_{30}$, which is the ratio of measured currents with an additional factor of 3 multiplying the $10-\mathrm{cm}$ (test case 1 ) data. It is then possible to solve for $K_{10}$ in terms of $\beta$.

$$
K_{10}=\frac{\beta-1}{1-\frac{\beta}{3}}
$$

If there were no end effect, it would be expected that $\beta=1$ and $K_{10}=0$. As a function of bias, we would expect $K_{10}$ to approach zero for low bias potentials and vary with $V_{a}^{0.5}$ given the above assumptions for variations of a cylindrical and spherical probe with bias.

Fig. 10 shows a comparison of experimental data and a possible theoretical variation to show slopes at the closest distances for the two test cases. The expected general character is seen but the variation with bias is somewhat larger than the expected $V_{a}^{0.5}$ behavior. The above derivation assumed that the end effect between the $10-$ and $30-\mathrm{cm}$ probes were identical, but it is possible that the $30-\mathrm{cm}$ probe did minimize end effects by placing the ends where the plasma density was lower (the density would also be varying along the probe itself). If so, then the shape of $K_{10}$ would be different than shown in Fig. 10. This remains to be analyzed. Nonetheless, these results do show end effects are a real concern that must be considered. In future experiments, we plan to use appropriate guards at each sample end, although the mechanical complexity for these small probes is considerable.

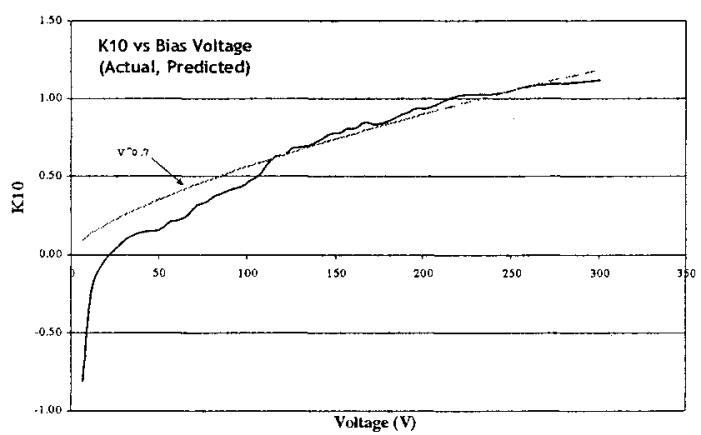

Fig. 10 Variation of predicted end effect currents to ideal cylindrical probe currents $\left(K_{10}\right)$ with bias voltage.

\section{Future Tests}

We are planning future experiments to help clarify and expand upon these initial results. First, we will be including a guard to mitigate against end-effects in all 
samples. We plan on more fully mapping and characterizing the plume and its energy distribution. We will be increasing the effective width of the tape samples to approximately $30 \lambda_{D}$. The geometries to be tested include cylinder, flat-ribbons of different widths, and ribbons with several levels of porosity. The porous ribbons are of two types: one type contains slots (different slot widths provide porosities of roughly 25,50 , and $75 \%$ ) and the second type contains a matrix of holes (different hole sizes all providing $50 \%$ porosity). The solid and porous ribbons will be oriented parallel and perpendicular to the plasma flow. These geometries represent possible candidate geometries for increasing tether lifetime over that predicted for a cylinder. Finally, we hope to be able to determine if turbulent effects are present in the near plasma as the bias is increased beyond the incoming ion beam energy.

\section{Conclusions}

We believe the experimental results to date in highspeed plasma indicate that the tape tether will be a viable option for bare-tether geometries. Widths well above a Debye length can be used, but we are not able to say yet if there is an experimentally determined maximum dimension (e.g., Sanmartín and Estes ${ }^{11}$ ).

The tape sample current collection appears to generally agree with equal-area cylinder OML model. However, there is definitely non-OML character also present in the data, especially for the $100-\mathrm{cm}$ case. Knees in the data appear just above the estimated ion beam energy range in all cases for the parallel orientation and the perpendicular case at the closest dimensions. It is also possible that at closest dimensions $(100 \mathrm{~cm})$, experimental data is not able to track OML theory at potentials starting around 5 times the ion beam energy (i.e., $200 \mathrm{~V}$ ). The differences between parallel and perpendicular orientation is also an example of high speed flow effect.

The possibility of end-effects even for our longer samples (test 2) does place some question on the overall quantitative assessment of the level of enhancement. In future experiments, a more sophisticated configuration can be used to minimize/eliminate this factor. However, we parenthetically note that adding an end collector may well be a positive enhancement for any bare EDT as our data suggests and suggested by others. ${ }^{7}$

\section{Acknowledgments}

The authors would like to thank the PEPL research group for assistance in performing these experiments. The authors thank Drs. R. Estes, N. Stone, and K. Wright for useful discussions about these results and their interpretation. This work was performed under contract from The Michigan Technic Corporation and NASA-MSFC.

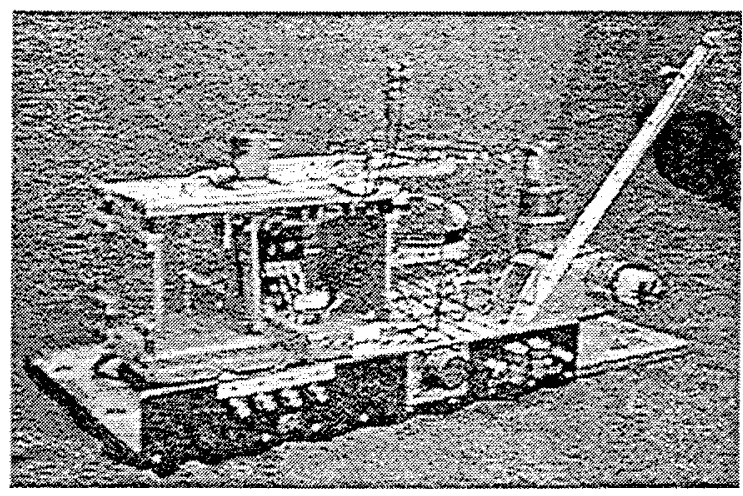

Fig. 11 Thrust stand with water-cooled shroud removed.

\section{Appendix A}

All thruster performance measurements were made with an inverted pendulum-type thrust stand based on the NASA LeRC/GRC design, the industry standard (see Fig. 11). The thruster was mounted to an aluminum plate that is connected to the core of a Lucas Schaevitz model 100-HR linear variable differential transformer (LVDT). The core resides within a LVDT coil that is mounted to the base of the thrust stand. Thus, the thrust stand measures the displacement of the aluminum plate due to engine thrust. Thruster/thrust-stand leveling is performed manually prior to chamber pump-down so that the LVDT core is in its null position within the coil. A remotely controlled stepper motor-driven pulley system is employed to provide in situ thrust-stand calibration by loading and off-loading small weights to simulate thrust. A linear curve fit of LVDT displacement versus thrust is then obtained and used for performance measurements. Soon after the thruster is turned off, a post-test calibration is performed. The springs of the stand are made with extra stiffness to minimize thruster deflection, thus allowing plume measurements to be made concurrently with performance measurements.

A bulk velocity model for the P5 was used to predict specific impulse and thrust as a function of thruster discharge voltage. The model uses two free parameters: $V_{l}$, a loss voltage associated with ionization and sheaths; and $\eta$, the fraction of the discharge current composed of electrons. In this model, the equation for specific impulse is given as

$$
I_{\mathrm{sp}}=\frac{1}{g} \sqrt{\frac{2 q\left(V_{d}-V_{l}\right)}{m_{i}}},
$$

and for thrust as

$$
F=I_{d}(1-\eta) \sqrt{\frac{2 m_{i}\left(V_{d}-V_{l}\right)}{q}} .
$$




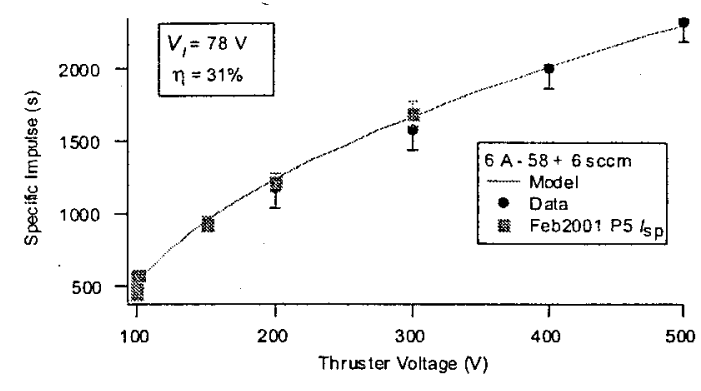

Fig. 12 Comparison of the P5 performance model with 1998 data from $\mathrm{Haas}^{5}$ (circles) and from Table 2 (squares).

A comparison of the model with P5 thrust data from $1998^{5}$ (circle) and from the recent test (squares) is given in Fig. 12. The model has excellent agreement with the data presented in Table 2, even at low voltage (i.e., below $200 \mathrm{~V}$ ).

\section{References}

${ }^{1}$ Sanmartín, J. R., Martinez-Snchez, M., and Ahedo, E., "Bare Wire Anodes for Electrodynamic Tethers," J. of Prop. and Power, Vol. 9, No. 3, 1993, pp. 353-360.

2 Johnson, Les, Gilchrist, Brian E., Estes, Robert D., Lorenzini, Enrico, and Ballance, Judy, "Propulsive Small Expendable Deployer System (ProSEDS) Space Experiment," AIAA Paper AIAA-98-4035, 1998.

${ }^{3}$ Forward, R. L., "Failsafe Multistrand Tether Structures for Space Propulsion," AIAA Paper AIAA-92-3214, 1992.

${ }^{4}$ Gallimore, A. D., Kim, S.-W., Foster, J. E., King, L. B. and Gulczinski III, F. S., "Near and Far Field Plume Studies of a One-Kilowatt Arcjet," AIAA J. Prop. Power, Vol. 12, 1996, pp. 105-111.

${ }^{5}$ Haas, James M., Gulczinski III, Frank S., Gallimore, Alec D., Spanjers, Gregory G., and Spores, Ronald A., "Performance Characteristics of a $5 \mathrm{~kW}$ Laboratory Hall Thruster," AIAA Paper AIAA-98-3503, 1998.

${ }^{6}$ Bilén, Sven G., Haas, James M., Gulczinski III, Frank S., Gallimore, Alec D., and Letoutchaia, Julia N., "ResonanceProbe Measurements of Plasma Densities in Electric-Propulsion Plumes," AIAA Paper AIAA-99-2714, 1999

${ }^{7}$ Dobrowolny, M., and Vannaroni, F., private communication, 1999.

${ }^{8}$ Stone, N. H., and Bonifazi, C., "The TSS-1R Mission: Overview and Scientific Context," Geophysical Research Letters, Vol. 25, No. 4, 1998, pp. 409-412 (and papers therin).

${ }^{9}$ Thompson, D. C., Bonifazi, C., Gilchrist, B. E., Willjams, S. D., Raitt, W. J., Lebreton, J.-P., Burke, W. J., Stone, N. H., and Wright, Jr., K. H., "The Current-Voltage Characteristics of a Large Probe in low Earth Orbit: TSS-1R Results," Geophysical Research Letters, Vol. 25, No. 4, 1998, pp. 413-416.

${ }^{10}$ Katz, I., Melchioni, E., Mandell, M., Oberhardt, M., Thompson, D., Neubert, T., Gilchrist, B., and Bonifazi, C., "Observations of Ionosphere Heating in the TSS-1 Subsatellite Presheath," J. Geophysical Research, Vol. 99, No. A5, 1993, pp. 8961-8969.

${ }^{11}$ Sanmartín, J. R., and Estes, R. D., "The Orbital-MotionLimited Regime of Cylindrical Langmuir Probes," Physics of Plasmas, Vol. 6, No. 1, 1999, pp. 395-405.

${ }^{12}$ Estes, R. D., and Sanmartín, J. R., "Cylindrical Langmuir Probes Beyond the Orbital-Motion-Limited Regime," Physics of Plasmas, Vol. 7, No. 10, 2000, pp. 4320-4325.
${ }^{13}$ Kruijff, M., van der Heide, E. J., De Venuto, F., Dobrowolny, M., Vannaroni, G., "Long Term Stability of Bare Conductive Tethers: Combined Results from Plasma Chamber Tests and Advanced Simulations," 2001 Space Technologies and Applications International Forum, 2001.

${ }^{14}$ Johannig, D., Seifert, W., and Best, A. "Analytic Density Correction for Cylindrical Langmuir Probes Showing End Effects, Plasma Physics and Controlled Fusion, Vol. 27, No. 2, 1985, pp. 159-179. 